\title{
The psychological impact of aesthetic procedures on the young patient
}

\author{
Ioana A Halip, Laura Gheucă Solovăstru, Laura Stătescu, Ioana A Popescu, \\ Adriana I Pătrașcu, Andra G Salahoru, Bogdan-Marian Tarcău, \\ Alina Stîncanu, Doinița Olinici Temelie, Dan Vâță
}

\begin{abstract}
Ioana-Alina Halip - M.D., Ph. D., Assistant Professor, Department of Dermatology, Faculty of Medicine, "Grigore T. Popa" University of Medicine and Pharmacy Iasi, Romania

Laura Gheucă Solovăstru - M.D., Ph. D., Professor, Department of Dermatology, Faculty of Medicine, "Grigore T. Popa" University of Medicine and Pharmacy Iasi, Romania. Senior Dermatovenerolog in Dermatology Clinic County Emergency Hospital "St. Spiridon" Iasi, Romania Laura Stătescu - M.D., Ph. D., Lecturer, Department of Dermatology, Faculty of Medicine, "Grigore T. Popa" University of Medicine and Pharmacy Iasi, Romania. Senior Dermatovenerolog in Dermatology Clinic County Emergency Hospital “St. Spiridon” Iasi, Romania

Ioana-Adriana Popescu - M.D., Ph. D. student, Department of Dermatology, of Medicine, "Grigore T. Popa" University of Medicine and Pharmacy Iasi, Romania.

Adriana I Pătrașcu - M.D., Ph. D. student, Department of Dermatology, Faculty of Medicine, "Grigore T. Popa" University of Medicine and Pharmacy Iasi, Romania. Senior Dermatovenerolog in Dermatology Clinic County Emergency Hospital "St. Spiridon” Iasi, Romania

Andra Georgiana Salahoru - Ph. D. student, Department of Psychology, "Alexandru Ioan Cuza" University, Iasi, Romania

Bogdan-Marian Tarcău - M. D., Dermatovenerology Clinic, "Sf. Spiridon" County Emergency Hospital Iasi, Romania

Alina Stîncanu - Senior Dermatovenerolog in Dermatology Clinic County Emergency Hospital "St. Spiridon" Iasi, Romania

Doinița Olinici Temelie - M.D., Ph. D., Lecturer, Department of Cell Biology, Faculty of Medicine, "Grigore T. Popa" University of Medicine and Pharmacy Iasi, Romania. Senior Dermatovenerolog in Dermatology Clinic County Emergency Hospital "St. Spiridon" Iasi, Romania Dan Vâță - M.D., Ph. D., Lecturer, Department of Dermatology, Faculty of Medicine, "Grigore T. Popa" University of Medicine and Pharmacy Iasi, Romania. Senior Dermatovenerolog in Dermatology Clinic County Emergency Hospital “St. Spiridon” Iasi, Romania
\end{abstract}

\section{ABSTRACT}

The field of aesthetic medicine is a trend in modern medicine and nowadays patients not only want to be healthy, they also want to enjoy life, be fit and minimize the effects of physiological 
s aging. Societal acceptance of aesthetic procedures is increasing and desire to improve the external appearance may be related to the extensive media coverage which basically increases the considered benefits. Young patients should understand that results of surgical aesthetical procedures may lead to permanent change. A complete assessment of reason why the young patient wishes to have the procedure, and what difference the patient thinks it will make to his/her life, should be undertaken. Poor psychological outcomes are possible and unrealistic expectations may should warn the doctor against procedure until the patient is emotionally mature and may need a psychological referral in order to maintain the psychosocial health and improve the quality of life.

\section{KEYWORDS:}

Aesthetic procedures, body dissatisfaction, psychological outcomes, quality of life, psychosocial health.

\section{INTRODUCTION}

What is beauty? The concept of beauty and attractiveness seems to be similar across cultures and religions and the characterization of a person as beautiful, is often based on some combination of inner beauty and outer beauty. Whereas inner beauty includes psychological factors such as personality, grace, intelligence and elegance, physical attractiveness refers to physical attributes which are valued on an aesthetic basis. Research shows that subjective attractiveness is largely biological and can be influenced by social trends and advertising especially in younger people. Moreover, studies which evaluated the consistency of physical attractiveness ratings across cultural groups showed that body attractiveness, and especially facial beauty is species-specific, not race-specific $(1,2)$.

Physical aspect and particularly the face is the public relations organ by excellence and may be considered the clock of age and the barometer of feelings. Health is the first thing that is considered indispensable in life and the second one is a pleasant appearance. Nowadays societal acceptance of aesthetic procedures is increasing and desire to improve the external appearance may be related to the extensive media coverage which

basically increases the considered benefits (3). A survey of American Society for Aesthetic Plastic Surgery shows that $53 \%$ of women and $49 \%$ of men approved of aesthetic surgery, while $27 \%$ of married Americans and $33 \%$ of young and unmarried Americans would consider it for themselves in the future (4).

The field of aesthetic medicine is a new trend in modern medicine and patients not only want to be healthy, they also want to enjoy life, be fit and minimize the effects of physiological aging. Indeed, patients are requesting non-invasive procedures with very little risk. Many of very valuable improvements of the face can be achieved by simple techniques and today many patients who want to improve their physical appearance are young or mature. Similarly, men and women are aware of such advances due to the media and their desire to improve physical appearance may become strong. Moreover, having a good look is considered a culture and it is even a need in many fields of activity.

Everybody at any age would like to have a pleasant aspect, without any blemish due to aging and aesthetic procedures are more 
accessible. Young people want to remain young and they want to correct the first signs of facial aging, to treat a minor defect or to enhance the beauty of a normal feature. In contrast with younger persons, senior patients do not want to change and very often want to have several simple procedures together instead of a major one (5).

\section{AESTHETIC PROCEDURES AND PSYCHIATRIC APPROACH}

Aesthetic procedures are an optional or medically unnecessary procedure requested by a patient to correct imperfections and improve appearance $(6,7)$. They are elective and they comprise all medical procedures that are aimed at improving the physical appearance and satisfaction of the patient, using non-invasive to minimally invasive procedures. Some aesthetic procedures are performed under local anaesthesia while some of them do not require it. Mainly, aesthetic procedures consist of:

- injections of neurotoxins (botox) and dermal fillers,

- chemical peels, microdermabrasion,

- body contouring and treatment of cellulite,

- skin nutrition,

- hair transplant or hair reduction,

- lifting, fat grafting,

- Platelet Rich Plasma (PRP),

- lasers and IPL (Intense Pulsed Light) treatments,

- scars management,

- venous treatment.

The real benefit of practicing aesthetic medicine is the type of care that practitioners are offering to their patients. Simple techniques for rejuvenation or associated with other minor surgical techniques give a natural look and not a postsurgical look, which is sometimes the result of a major procedure. The satisfaction of the patient may be the same as surgical treatment of skin neoplasia or tumors' pathology of severe photo damage in senior persons $(8,9,10)$.

The largest age group of patients who demand aesthetic procedures (surgical and nonsurgical) is between 35 and 50 years (11). However, in 2016 statistical data from American Society for Aesthetic Plastic Surgery show that $25.3 \%$ belongs to group 19-34 years and $25.6 \%$ belongs to older age, respectively $35-50$ years (11). These results may be related to the increasing societal acceptance of aesthetic surgery. Furthermore, may be the result to the obsession of popular media with body image which increases the focus on such procedures, particularly in the younger age group with internet navigation. But how young is too young for aesthetic procedures? The number of teenage patients who are requiring such dermatologic treatment is small and is reported that only $1.8 \%$ of all patients undergoing surgical procedures in 2016. American Society for Aesthetic Plastic Surgery indicated in data from the last 16 years similar rates every year over the time period, ranging between 1 and $3 \%$ (11).

Food and Drug Administration (FDA) reported that the greatest concern is that the teen will have unrealistic expectations. Clearly, the doctor should assess the emotional and physical maturity of younger patients before any invasive procedures. It is mandatory that the young patient should understand that results of surgical aesthetical procedures may lead to permanent change. A complete assessment of reason why the young patient wishes to have the procedure, and what difference the patient thinks it will make to his/her life, should be undertaken. Unrealistic expectations may should warn the 


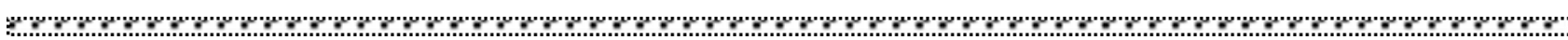

doctor against procedure until the patient is emotionally mature and may need a psychological referral (11).

Unfortunately, the ideal patient (young or senior) who is referring to dermatologist for aesthetic procedure does not exist. The ideal patient should be able to develop discussions and show understanding, having effective communication skills. Moreover, he should be pleasant to the medical staff, well educated, and especially well informed regarding the aesthetical treatment. The ideal patient has realistic expectations of the outcome and a full understanding of potential complications, being compliant with pre and post procedure instructions.

On the one hand, aesthetic procedures improve the quality of the patients' lives after they accepted the good results of procedures. On the other hand, it was also reported that some patients were considered to have psychological problems, and the morbidity rate was reported to be higher (12). Young patients may have social functional disturbance, and a considerable number of these patients may seek aesthetic procedures rather than ask psychiatrists for help. Anxiety, depression, obsessive-compulsive disorder, body dysmorphic disorder, dependent personality disorder may be identified according to psychological examination. These psychological disorders might lead to a poor surgical outcome and high risk of dissatisfaction, and even recurrent surgical interventions (12). This is the reason why is necessary to understand the psychological status of every person seeking aesthetic surgical or nonsurgical procedures, and patients' selection is considered to be the first factor that determines the success or failure of it (13).
In a cross-sectional study (14) which included 315 patients with an average age of $30 \pm 8.4$ years admitted to the Department of Plastic Surgery in a General Hospital in China from July 2016 to July 2017, investigated the prevalence and risk factors in preoperative patients who visited a aesthetic plastic surgery clinic. The results indicated that the preoperative anxiety was more obvious than depression. Also, education level was associated with the diagnosis of anxiety ( $\mathrm{p}=$ $0.034)$ while education level $(\mathrm{p}=0.001)$ and occupation $(\mathrm{p}=0.015)$ were associated with the diagnosis of depression (14).

Gillen (15) investigated that subjects with greater positive body image had less depression, higher self-esteem, a smaller percent of unhealthy dieting behaviours and greater intentions to protect their skin from sun exposure and photo damage and these associations were not correlated with gender (15). Therefore, Gillen concluded that healthcare providers should promote positive body image because of its potential health benefits (15). Similarly, other investigators $(16,17)$ provided a great impact of aesthetic surgery (breast augmentation or reduction, abdominoplasty and facial procedureblepharoplasty, rhinoplasty, lipoplasty or scar correction) on body image with a connection between aesthetic procedures and physical and mental health.

Physical appearance is an integrative part of personal identity and its relation to individuals' self-perception begins early in life (18). The socio-cultural environment may influence perceptions of attractiveness (19). Tripartite Influence Model (20) sustain that attractiveness ideals are transmitted by three primary sociocultural influences: parents, colleagues and the media. A research about influence of traditional media such as television programs, adverts or newer types of 
media (social networking sites) on women's consideration of aesthetic surgery enrolled 118 women aged 18-29 years (20). The participants were divided in two arms. One arm received images from social networks with young adult women who had rhinoplasty, soft tissue fillers, Botox, laser skin resurfacing and microdermabrasion using an Analogue Visual Scale in order to evaluate satisfaction (20). The second arm received for evaluation 30 travel images. Results showed that participants who had seen images of enhanced females revealed a tendency for an increased desire for aesthetic surgery compared to participants who saw images of travel. Moreover, the relationship between social media use and desire for aesthetic surgery was not mediated by body dissatisfaction. The appearance pressures from social media may lead people who are more involved in their appearance to desire changing strategies such as aesthetic procedures and therefore may have greater expectations from therapies (20). In this way, screening measures should be used before aesthetic treatments in order to assess better patients' motivations and their psychological functioning. It is likely that aesthetic treatments will have negative outcomes if their motivations were based on resembling someone they have seen on social media (21).

Other studies $(22,23)$ have shown a connection between body dissatisfaction and acceptance of aesthetic surgery among women, suggesting that people may consider aesthetic treatment as a method to obtain psychological benefits such as higher selfesteem and social benefits becoming more attractive. Self-compassion has positive (mindfulness, common humanity, selfkindness) and negative (over-identification, isolation, self-judgment) components. 220 young Italian women with age between 19 and 31 years, completed a questionnaire with reference to the fields of interest (24). It was examined the association between selfcompassion components and body dissatisfaction and acceptance of aesthetic surgery among women and physical appearance comparison. Analysis revealed that higher mindfulness was directly associated to lower acceptance of aesthetic surgery. Common humanity and self-kindness were connected to acceptance of aesthetic surgery for social reasons (24). However, other experimental studies claimed that even if their body dissatisfaction had decreased, body image quality of life and self-esteem have not increased in women who had received aesthetical procedures (25).

Overall, systematic reviews after 1950 which have analysed the impact of aesthetic procedures showed mixed results probably due to variation in the procedures or to the methodological quality of life used in studies. Some of them revealed satisfaction, selfesteem and improved psychological distress following aesthetical procedures (7, 26), others showed few positive long-term psychological effects $(27,28)$. Predictors of poor outcomes included clinically diagnosed disorders with a psychological component like depression or body dysmorphic disorder, but they may also include non-clinically diagnosed disorders such as anxiety, low selfesteem and social parameters like age and relationship status (29).

In a review of 37 studies (29), factors as being young, being male, having unrealistic expectations of the procedure or previous unsatisfactory aesthetic surgery, minimal deformity or motivation based on relationship issues were associated with poor psychosocial outcome. Aesthetical surgical procedures were associated with both intimate partner violence and verbally abused women, smoker 


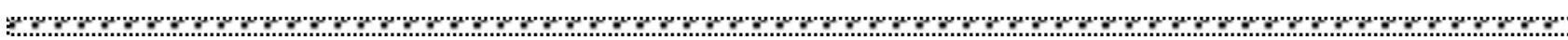

or people taking medication for anxiety, nervous conditions or sleep disorders (30).

A review of nine studies studied the effect of aesthetic interventions on post procedure psychological outcomes (31). Various types of aesthetic procedures such as botulinum toxin type A injection and facelift were included. Facelift was associated with improvement of self-esteem and quality of life/social functioning, but had no positive effects of anxiety or depression $(26,29)$. The impact on psychosocial health of aesthetic procedure such as hyaluronic acid fillers and botulinum toxin type $\mathrm{A}$ remains however poorly defined.

Body dysmorphic disorder represents the disorder which is characterized as a preoccupation with a slight or no observable defect in appearance and is associated with obsessive thinking and compulsive behaviours and which leads to a disruption in activities of daily life. This psychiatric condition is likely of greatest relevance to the aesthetic doctor (32). It occurs in approximately $1 \%$ to $2 \%$ of the general population and is estimated equally across women and men (33). Patients with this disorder may present concern with any feature of the body such as facial skin, nose, and hair. They are often convinced that they will feel better if they change their physical appearance and therefore they are seen frequently in aesthetic medical practices. The majority of studies of persons with body dysmorphic disorder unfortunately suggested that greater than $90 \%$ of patients reported no change or a worsening of their symptoms following an aesthetic treatment. Some of them reported that the level of preoccupation with the given feature was not improved and others indicated that they became concerned about another aspect of their physical appearance (33).
Data (33) suggests that many providers have identified patients with this condition during the preoperative consultation and refused with the required treatment. Specific questions should be prepared to assess for the presence of body dysmorphic disorder. Examples of relevant questions may include the following:

1. Is the patient preoccupied with the perceived physical defect?

2. Does this preoccupation cause clinically significant distress like anxiety, depression or hopelessness?

3. Does the preoccupation with physical defect cause clinically significant impairment in functioning like difficulty with or avoidance of family, school, social or work activities?

Analysing patients answers, those individuals with no or minimal physical defect who experience resulting clinically significant distress or impairment in functioning and who perform at least one associated repetitive behaviour are likely to have body dysmorphic disorder. Due to the safety risks for both patients and aesthetic surgeons or dermatologists and the evidence that aesthetic treatment outcomes among people are poor, body dysmorphic disorder is considered a contraindication for aesthetic treatments.

Saying "no" to a patient can be difficult but is sometimes necessary. You should listen to your intuition and be cautious. If you have concerns, do not offer the patient aesthetic procedure. So when to say "no"? The aesthetic doctor may consider the patient unsuitable for several reasons. According to Gorney and Martello, the patient may be either anatomically or psychologically unsuitable for the procedure (34). From an anatomic viewpoint, the feature that the patient wishes to have corrected must be visible to the surgeon and able to be corrected. The ideal patient fits around the 
diagonal of Gorney's patient selection graph (35).

Lately, young women are being encouraged to embrace and accept the way that they look, and this may help to avoid aesthetic procedures. For example, the number of aesthetic procedures conducted in 2016 fell $40 \%$ from a record-breaking high in 2015 (36). In this manner, the decreased societal pressure for an idealized beauty standard may be encouraging women away from having aesthetic procedures (37). Psychological assessment of young women who are interested in aesthetic surgical or nonsurgical procedures is highly recommended $(38,39)$.

Aesthetic surgery does not necessarily help young people to improve their body image
$(25,33)$. When women seek aesthetic surgery without changing their attitude toward the self, is a great chance that they will have low self-esteem also after undergoing surgery. This fact could lead them to look for further procedures, without ever feeling comfortable with their body. Surgeons and dermatologists might recommend alternative strategies, such as self-compassion trainings, that could help women to change the way they relate to their body image. Optimal levels of common humanity and mindfulness could decrease women's acceptance of aesthetical procedures as well, at least for psychosocial health. The existence of self-kindness and acceptance of aesthetic procedures should be further explored before considering the advantages and disadvantages of trainings that specifically focus on this dimension.

\section{CONCLUSIONS}

It is well known that minor imperfections of the skin or aesthetic anomalies can cause much distress. Prevention is better than cure and mini invasive aesthetic techniques are frequently indicated. In young patients simple procedures are very often enough. Aesthetic procedures today is not only rejuvenation but it is also maintenance of youth and improvement of the mind. It is not rare the situation when the social media and he publicity creates in the public's mind an image that is unreal and deceptive and may affect peoples psychosocial health.

In aesthetic medicine, it is necessary to know how and also why a procedure has to be selected. All the problems of patient selection should be discussed as well as minor surgical details about all the procedures used for physical appearance improvement of region of the face, neck, and scalp, including also peelings, biological fillers, short access facial elevation or lifting, mesotherapy techniques or removal of the fat.

Understanding the patients' motivations for aesthetic interventions and their expectations of the outcomes are the keys to achieving satisfied patients. Vulnerable patients should be identified and given appropriate care. Doctors should explore with patients the reasons for their request for aesthetical procedures using validated screening tools that look at psychosocial issues in addition to body image disorders. Practitioners should be aware of reasons underlying requests and of those who are at risk of poor outcomes. Moreover, the dermatologist should require a full patient education including discussions about the benefits and the possible risks. Favourable and unfavourable results to aesthetic procedures should be considered and fully discussed. Similarly, a second consultation after an aesthetic procedure is almost always necessary, with post procedure follow-up including detailed written instructions for the recovery. Those patients with 

unsatisfactory outcomes should be seen more often to improve communication in order to evaluate the psychological outcomes.

\section{ACKNOWLEDGEMENTS AND DISCLOSURES}

The authors state that they are no declared conflicts of interest regarding this paper.

\section{REFERENCES}

1. Bashour M. History and current concepts in the analysis of facial attractiveness. Plast Reconstr Surg 2006; 118:741-756.

2. Cunningham MR, Roberts AR, Barbee AP, et. al. "Their ideas of beauty are, on the whole, the same as ours": consistency and variability in the cross-cultural perception of female physical attractiveness. J Pers Soc Psychol 1995; 68:261-279.

3. Lee S-Y. The effect of aesthetic surgery realty shows on women's beliefs of beauty privileges, perceptions of aesthetic surgery, and desires for aesthetic enhancements. Am Commun J 2014; 16:1-14.

4. http://www.surgery.org/sites/default/files/Stats2010_1.pdf.

5. Shiffman MA, Mirrafati SJ, Lam SM (2007). Simplified Facial Rejuvenation, 2008th ed.; Springer: Berlin Heidelberg, Germany; pp:46-69.

6. Rohrich RJ. The American Society of Plastic Surgeons' procedural statistics: what they really mean. Plast Reconstr Surg 2003;112:1389-1392.

7. von Soest T, Kvalem IL, Wichstrom L. Predictors of aesthetic surgery and its effects on psychological factors and mental health: a population-based follow-up study among Norwegian females. Psychol Med 2012;42:617-626.

8. Gheucă Solovăstru L, Vâță D, Stîncanu A, Ciubara AM, Andrese E. The pshychosocial impact on patients with skin neoplasia. Bulletin of Integrative Pshychiatry 2013;3(58):33-38.

9. Grajdeanu IA, Gheuca Solovastru L, Vata D et al. Improving the quality of life of patients by using imaging techniques. Bulletin of Integrative Psychiatry 2019; (80)1:43-48.

10. Gheucă Solovăstru L, Vâță D, Stătescu L, Constantin MM, Andrese E. Skin cancer between myth and reality, yet ethically constrained. Rev Rom Bioet 2014; 2(12): 47-52.

11. http://www.surgery.org/sites/default/files/ASAPS-Stats2016.pdf

12. Herruer JM, Prins JB, van Heerbeek N, Verhage-Damen GW, Ingels KJ. Negative predictors for satisfaction in patients seeking facial aesthetic surgery: a systematic review. Plast Reconstr Surg 2015;135:1596-1605.

13. Andretto Amodeo C. The central role of the nose in the face and the psyche: review of the nose and the psyche. Aesthet Plast Surg 2007;31:406-410.

14. Wei L, Ge C, Xiao W, Zhang X, Xu J. Cross-sectional investigation and analysis of anxiety and depression in preoperative patients in the outpatient department of aesthetic plastic surgery in a general hospital in China. J Plast Reconstr Aesthet Surg 2018;71(11):1539-1546.

15. Gillen MM. Associations between positive body image and indicators of men's and women's mental and physical health. Body Image 2015;13:67-74.

16. Cingi C, Songu M, Bal C. Outcomes research in rhinoplasty: body image and quality of life. Am J Rhinol Allergy 2011; 25(4):263-267.

17. Litner JA, Rotenberg BW, Dennis M, Adamson PA. Impact of aesthetic facial surgery on satisfaction with appearance and quality of life. Arch Facial Plast Surg 2008;10(2):79-83.

18. Davison KK, Birch, LL. Weight status, parent reaction, and self-concept in five-year-old girls. Pediatrics 2001;107(1):46-53.

19. Cafri G, Yamamiya Y, Brannick M, Thompson, JK. The influence of sociocultural factors on body image: A meta-analysis. Clinical Psychology:Science and Practice 2005;12, 421-433.

20. Shome D, Vadera S, Male SR, Kapoor R. Does taking selfies lead to increased desire to undergo aesthetic surgery. J Cosmet Dermatol 2020;19(8):2025-2032.

21. Lee HH, Damhorst ML, Paff OJ. Body satisfaction and attitude theory: Linkages with normative compliance and behaviors undertaken to change the body. Fam Consum Sci Res J 2009; 37(4):466-488. 
22. Lunde C. Acceptance of aesthetic surgery, body appreciation, body ideal internalization, and fashion blog reading among late adolescents in Sweden. Body Image 2013; 10: 632-635.

23. Markey CN, Markey PM. Correlates of young women's interest in obtaining aesthetic surgery. Sex Roles 2009;61:158-166.

24. Nerini A, Matera C, Di Gesto C, Policardo GR, Stefanile C. Exploring the Links Between Self-Compassion, Body Dissatisfaction, and Acceptance of Aesthetic Surgery in Young Italian Women. Front Psychol 2019; 10:2698.

25. Sobanko JF, Dai J, Gelfand JM, Sarwer DB, Percec I. Prospective cohort study investigating changes in body image, quality of life, and self-esteem following minimally invasive aesthetic procedures. Dermatol Surg 2018; 44:1121-1128.

26. Shridharani SM, Magarakis M, Manson PN, Rodriguez ED. Psychology of plastic and reconstructive surgery: a systematic clinical review. Plast Reconstr Surg 2010;126:2243-2251.

27. Cook SA, Rosser R, Salmon P. Is aesthetic surgery an effective psychotherapeutic intervention? A systematic review of the evidence. J Plast Reconstr Aesthet Surg 2006; 59:1133-1151.

28. Sarwer D. The psychological aspects of aesthetic breast augmentation. Plast Reconstr Surg 2007;120 (1):110117.

29. Honigman RJ, Phillips KA, Castle DJ. A review of psychosocial outcomes for patients seeking aesthetic surgery. Plast Reconstr Surg 2004; 113:1229-1237.

30. Schofield M, Hussain R, Loxton D, Miller Z. Psychosocial and health behavioural covariates of aesthetic surgery: Women's Health Australia study. J Health Psychol 2002;7:445-447.

31. Brunton G, Paraskeva N, Caird J, Bird KS, Kavanagh J, Kwan I et al. Psychosocial predictors, assessment, and outcomes of aesthetic procedures: a systematic rapid evidence assessment. Aesthetic Plast Surg 2014;38(5):1030-1040.

32. Crerand CE, Franklin ME, Sarwer DB. Patient safety and body dysmorphic disorder in aesthetic surgery patients. Plast Reconstr Surg 2008;122:1-15.

33. Sarwer DB, Pruzinsky T, Cash TF, Goldwyn RM, Persing JA, Whitaker LA (2006). Psychological aspects of reconstructive and aesthetic plastic surgery. Lippincott Williams \& Wilkins: Philadelphia; pp:267-283.

34. Gorney M, Martello J. Patient selection criteria. Clin Plast Surg 1999; 26:37-40.

35. Gorney M. Mirror, mirror on the wall: the interface between illusion and reality in aesthetic surgery. Facial Plast Surg Clin North Am 2008;16: 203-205.

36. https://baaps.org.uk/media/press_releases/29/the bust_boom_busts

37. Henderson-King D, Henderson-King E. Acceptance of aesthetic surgery: scale development and validation. Body Image 2005; 2: 137-149.

38. Mulkens S, Bos AE, Uleman R, Muris P, Mayer B, Velthuis P. Psychopathology symptoms in a sample of female aesthetic surgery patients. J Plast Reconstr Aesthet Surg 2012; 65: 321-327.

39. Naami A, Salehi HM. Prediction of aesthetic surgery tendency based on mindfulness, personality dimensions, perfectionism and mental health components. Int J Sch Health 2016;3:e32746.

\section{Correspondence}

Laura Gheucă Solovăstru,

M.D., Ph. D., Professor, Department of Dermatology, Faculty of Medicine, "Grigore T. Popa"

University of Medicine and Pharmacy Iasi, Romania

Adress: Dermatology Clinic County Emergency Hospital “St. Spiridon”, Bd. Independenței no. 1, Iasi, Romania, lsolovastru13@yahoo.com

Submission: 10may2021

Acceptance: 27 jul2021 